\title{
Energía del futuro: \\ Bioalcoholes a partir de Residuos Sólidos Urbanos (RSU)
}

The energy of the future:

Bioalcuhols from urban

solid residuals (USR)

José Alejandro Martínez. *

Nancy Montoya*

María Sierra ${ }^{\star \star *}$

L'énergie de l'avenir :

les alconls biologiques élaborés

à partir des Résidus Salides

Municipaux (DSM)

Energia do futuro: Bioálcod

a partir de Resíduos

Súlidos Lrbanos (RSL)

"Ingeniero Químico con Maestría en Ingeniería Ambiental; Doctorando en Administración estratégica de negocios, de la Pontificia Universidad Católica de Perú; Doctorando en Business Administration, del Maastricht School of Management

(Holanda); especialista en manejo de residuos y diplomado en docencia, en educación ambiental y en gestión sostenible.

** Ingeniera de Saneamiento y Desarrollo Ambiental.

Universidad Católica de Manizales,

Especialista en Gerencia de Proyectos, Universidad EAN;

Especialista en Gestión de Residuos Sólidos y Suelos Contaminados. Universidad Castilla de La Mancha (UCLM).

${ }^{* * *}$ Maestría en Ciencias de Energías, Tecnología Medioambiental y Economía.

City University London;

Especialista en Finanzas y Negocios Internacionales, acreditada internacionalmente por la ACBSP,

Universidad EAN;

Diplomado en Administración de Riesgos y Auditoría Forense (120 horas)

Pontificia Universidad Faveriana.

Administradora de Empresas,

Universidad EAN. 


\section{RESUMEN}

El presente artículo tiene como objetivo presentar un panorama ambiental, técnico y económico de la producción de bioalcohol, a partir de la Fracción Orgánica de Residuos Sólidos Urbanos (FORSU), tema que ha sido trabajado en investigaciones conjuntas con la Universidad de Castilla La Mancha (UCLM-España) y complementado con otros desarrollos realizados a través de la línea de investigación en Residuos Sólidos de la Universidad EAN, con el apoyo de estudiantes de especialización tanto en gestión de residuos sólidos, como en finanzas y negocios internacionales.

El aprovechamiento no convencional de los Residuos Sólidos Urbanos (RSU), y en particular, para las condiciones de América Latina en donde más del 50\% de los RSU corresponden a residuos de características orgánicas, termina siendo una posibilidad para agregar valor, generar crecimiento económico y crear condiciones de trabajo dignas para las personas vinculadas al aprovechamiento informal de residuos. Por ello, aprovechando el potencial de la biotecnología que ofrece diversas opciones para la generación de energías renovables, se busca abordar la producción de bioetanol a partir de procesos biológicos de metabolización de la materia orgánica mediante fermentación.

En este artículo se presentará inicialmente, el contexto global de la producción de biocombustibles en Colombia, para posteriormente presentar el desarrollo de una fase preliminar a escala de laboratorio para la obtención de bioalcohol mediante una unidad de fermentación anaerobia experimental. Posteriormente se mencionarán los elementos económicos y financieros relativos a la implementación de este tipo de tecnologías y que por consiguiente podrían afectar el desarrollo a escala industrial de dicho proceso. Los resultados permitirán evaluar la viabilidad técnica y económica, al menos en una etapa preliminar, de implementación de este tipo de procesos en Colombia.

\section{ABSTRACT}

In this research a computational simulation of the process of anaerobic digestion was carried out, using a homogenous mixture of bovine manure and citric residuals to assess the viability of renewable energy generation from the co- digestion of these residuals. In accordance with the former outcomes, an experimental stage was carried out in which periodic measurements of the control variables of anaerobic biodegradability were taken such as $p H$, alkalinity, oxygen chemical demand (total and soluble), among others. Also, process performance parameters were established and comparative values between experimental outcomes and computational results were determined.

Additionally, a simulation of the anaerobic digestion process was carried out under similar conditions formerly established in a previous research through different experiments using the ADM-1 model. Based on the expected outcomes and as a result of experiments and simulations, the model precision was evaluated taking into account the values obtained from experimental measurements. Finally, a simplified model was implemented to evaluate energy production by a pilot- scale installation which performs the anaerobic treatment of organic residuals under observation. This model showed that there was an energetic deficit at the beginning; however, once the biogas production started the process, it became autosustainable and the system provided an increasing amount of available energy.

\section{Palabras clave}

Residuos Sólidos Urbanos (RSU)

Bioetanol Aprovechamiento

Fermentación anaerobia Biocombustibles
Urban solid residuals (USR) Bioethanol

Achievement Anaerobic fermentation Biofuels 


\section{RESUMÉÉ}

Dans cet article nous avons simulé par ordinateur le processus de digestion anaérobique d'un mélange homogénéisé de fumier bétail et de résidus d'agrumes afin d'évaluer la viabilité des énergies renouvelables à partir de la transformation des déchets. Sur la base des résultats obtenus et grâce au développement de la phase expérimentale lors de laquelle étaient mesurées de façon périodiques les variables de contrôle du processus de biodégradation anaérobique tels que le pH, l'alcalinité, la demande chimique en oxygène (total et soluble), nous avons établi les performances des paramètres du processus et déterminé des valeurs pour la comparaison entre résultats expérimentaux et résultats informatiques.

Nous avons en outre réalisé une simulation du processus de digestion anaérobique dans des conditions similaires à celles précédemment établis par d'autres essais expérimentaux à travers la mise en cuvre du modèle ADM-1. Grâce aux résultats obtenus par le biais de simulations et mesures expérimentales, nous avons évalué la précision du modèle concernant les valeurs des mesures expérimentales. Un modèle simplifié pour l'évaluation de l'énergie produite par une installation à échelle réduite qui effectue le traitement anaérobique des résidus organiques a finalement été mis en place. Le modèle a montré qu'il existe au départ un déficit énergétique pour que le processus se réalise, mais une fois la production de biogaz enclenchée, le processus devient autonome et le système fournit une quantité croissante d'énergie disponible.

\section{RESUMO}

Nesta pesquisa foi feita a simulação computacional do processo de digestão anaeróbica da mistura homogeneizada de esterco bovino e resíduos cítricos com o propósito de avaliar a viabilidade da geração de energia renovável a partir da co-digestão destes resíduos. Baseados nos resultados obtidos anteriormente, e mediante o desenvolvimento da etapa experimental na qual foram realizadas medições periódicas das variáveis de controle do processo de biodegradabilidade anaeróbica tais como $\mathrm{pH}$, alcalinidade, demanda química de oxigênio (total e solúvel), entre outras, estabeleceram-se os parâmetros de desempenho do processo e foram determinados os valores de comparação entre os resultados experimentais e os resultados computacionais.

Adicionalmente, realizou-se a simulação do processo de digestão anaeróbica em condições semelhantes às estabelecidas previamente em outra investigação a partir de ensaios experimentais, através da implementação do modelo ADM-1. Baseados nos resultados obtidos através das medições experimentais e as simulações, a precisão do modelo foi avaliada com respeito aos valores das medições experimentais. Finalmente, implementouse um modelo simplificado para a avaliação da energia produzida por uma instalação a escala piloto que realiza o tratamento anaeróbico do resíduo orgânico em estudo. O modelo mostrou que inicialmente existe um déficit energético para que o processo ocorra; contudo, uma vez a produção de biogás começa, o processo se torna autosustentável e o sistema entrega uma quantidade crescente de energia disponível.

\section{Mots clefs}

\author{
Déchets solides \\ municipaux (DSM) \\ Utilisation \\ Fermentation anaérobique \\ Bioéthanol \\ Biocarburants
}

\section{Palavras-chave}

Resíduos sólidos urbanos

(RSU)

Bioetanol

Aproveitamento

Fermentação

Anaeróbia

Biocombustiveis 


\section{INTRODUCCIÓN}

$O^{i}$ bien la implementación de la Política Nacional de Producción y Consumo Colombiana y de la Política Nacional de Gestión Integral de Residuos Sólidos, ha permitido que el país haya "...llegado a avances como el que actualmente el $79 \%$ de los municipios del país dispongan sus residuos en sitios adecuados, tales como rellenos sanitarios y plantas integrales, siendo este porcentaje equivalente a 25.091 toneladas por día), así como el cierre preventivo y definitivo de sitios temporales de disposición en el territorio nacional que habían sido autorizados (o no) por la entidad competente respectiva..." (Martínez, 2012), es claro que se requiere de opciones para reducir la cantidad de residuos que van a los rellenos de paso, aprovechar su potencial.

El objetivo es investigar el proceso de producción de bioetanol y sus características durante la transformación de la materia orgánica, con miras al aprovechamiento no convencional de este material. En la actualidad, la materia orgánica se usa en la producción de compost o de humus a través de lumbricultivos, sin embargo, deben buscarse otras alternativas que permitan generar nuevos emprendimientos alrededor del tema, con resultados financieros visibles y una producción a gran escala.

Las características de uso de biocombustibles y sus normas en Colombia, implican la necesidad de contener el 10\% como mínimo de etanol en la gasolina motor, con proyecciones de aumento a través del tiempo. El mercado objetivo principal corresponde al sector de la industria de los biocombustibles y los centros mayoristas de mezcla, que actualmente requieren mezclar diferentes concentraciones de etanol con el combustible fósil para motores. En Colombia, la Ley 693 de 2001 establece las características de uso de biocombustibles y los requerimientos del contenido de alcohol etanol en la gasolina motor. Actualmente, el país es el segundo productor de etanol en Latinoamérica después de Brasil, sin embargo, la materia prima compite fuertemente con el sector de alimentos a nivel mundial lo que genera controversias de carácter técnico, social y ético.

El tema de las energías alternativas, ha generado una gran expectativa en cuanto a las oportunidades que se tienen de aprovechar los residuos sólidos orgánicos, generar alto impacto ambiental, económico y social, e impulsar el uso y desarrollo de los biocombustibles. La propuesta de innovación es generar bioalcohol como una energía alternativa a partir de residuos que actualmente se desperdician y que pueden generar impactos negativos a nivel ambiental y social, además de no competir con productos vegetales que sirven tanto como alimento como para la producción de biocombustibles.

El presente artículo busca, a partir de la evaluación preliminar de una propuesta de innovación, generar bioalcohol a partir de residuos que actualmente se desperdician y generan alto impacto negativo ambiental, avanzar en el análisis de la posible implementación de esta tecnología en el entorno colombiano, su viabilidad financiera y sus beneficios en términos sociales, ambientales, de seguridad alimentaria y de creación de valor para el desarrollo del país. 


\section{CONTEXTO DE LA MATERIA PRIMA: LOS RESIDUOS SÓLIDOS URBANOS (RSU)}

H oy en día, como menciona Martínez, (2012) y Martínez, (2013), cerca de 3.000 millones de habitantes generan en promedio $1.2 \mathrm{~kg}$ por persona y por día de residuos sólidos urbanos (RSU), para una generación mundial total de cerca de 1.300 millones de toneladas por año. De esa cantidad, América Latina genera cerca de 160 millones de toneladas por año. La composición de los RSU varía en todo el mundo, de región a región, algo similar a lo que ocurre con el índice de generación de RSU per cápita; en particular, en América Latina el último informe del Banco Mundial estima que la fracción orgánica de los RSU son un $54 \%$ del total, el papel tiene el $16 \%$ y el plástico el $12 \%$, correspondiendo esta distribución a valores de países pobres y de ingresos medios, según menciona Betancourt; Martínez (2012).

El manejo de los residuos sólidos podría llegar a convertirse entonces, en un problema ambiental para las ciudades, dado el proceso de urbanización que a nivel mundial se viene presentando en los últimos 20 años. En Colombia, de las más de 25.000 toneladas por día que se generan de Residuos Sólidos Urbanos (RSU), solo Bogotá produce más del $26 \%$, esto es, 6.600 toneladas

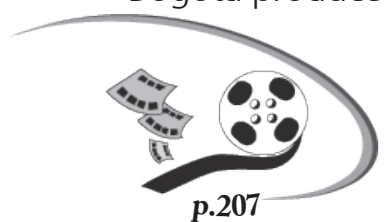
diarias "...las cuales se disponen en el Relleno Sanitario Doña Juana (opción centralizada), adicional a las 1.200 toneladas día aproximadamente que no van a disposición final sino que se desvían a través de opciones no centralizadas de aprovechamiento (cooperativas de recicladores, opciones privadas de manejo postproducción, etc.)..." (Martínez, 2012). Los RSU de la ciudad de Bogotá se disponen de manera mezclada, dañando el potencial de aprovechamiento de algunos materiales y agotando su capacidad y vida útil, con graves inconvenientes a nivel ambiental y social. De esa cantidad que se dis-pone diariamente en la ciudad, más del $65 \%$ corresponde a residuos orgánicos, lo cual es una cifra suficientemente alta como para interesar a autoridades locales y a inversionistas privados.

Los Residuos Sólidos Urbanos (RSU), poseen características físicas y químicas que si se presenta un inadecuado manejo después de su generación, pueden provocar problemas de salubridad y deterioro al ambiente. Los efectos más frecuentes generados por el inadecuado manejo de los RSU se presentan a continuación:

\subsection{Contaminación atmosférica}

La fermentación de la materia orgánica en ausencia de oxigeno genera metano, el cual es un gas de efecto invernadero que es 25 veces más potente que el dióxido de carbono (según los análisis del Protocolo de Kioto), siendo este gas metano ( $\mathrm{CH} 4$ ) el que constituye el $50 \%$ de las emisiones gaseosas producidas en los rellenos sanitarios.

\subsection{Contaminación edáfica}

Las propiedades físicas, químicas y biológicas del suelo se ven alteradas cuando sobre este, se depositan residuos no biodegradables.

\subsection{Contaminación de las aguas superficiales o subterráneas}

Los líquidos liberados por descomposición de la fracción orgánica de los residuos, sumados a la percolación de la lluvia por un mal cubrimiento del sitio de disposición, genera que sean arrastrados compuestos orgánicos del material dispuesto, fenómeno que recibe el nombre de lixiviación. 


\subsection{Afectación por olores}

Formadas por la descomposición de la materia orgánica presente en gran parte de los Residuos Sólidos Urbanos, principalmente el H2S, que provocan una serie de malos olores.

\subsection{Proliferación de plagas y aparición de focos infecciosos}

Cuando se acumulan de forma incontrolada los RSU, se favorece la proliferación de plagas como roedores e insectos y también la proliferación de aves carroñeras, entre otros, vectores que pueden ser origen de focos de infección.

La gestión integral de los Residuos Sólidos Urbanos (RSU), bajo la normatividad colombiana vigente, comprende las actividades desde la generación, recolección, transporte, almacenamiento, separación y disposición final de los RSU. En general, los componentes de la Fracción Orgánica de los Residuos Sólidos Urbanos (FORSU), pueden ser transformados con el fin de obtener, a partir de ellos, nuevos productos con otras aplicaciones competitivas como por ejemplo, los procesos de biometanización y compostaje, técnicas mediante las cuales se pueden obtener fuentes generadoras de energía que pueden usarse como combustible alternativo (para el primer caso) o productos de valor agregado con bajo impacto ambiental (en el segundo). Otros procesos que pueden encargarse de aprovechar energéticamente los RSU (incluyendo otras fracciones diferentes a la orgánica), son la gasificación, la hidrogenación, la pirólisis y la oxidación, muy usadas en los países desarrollados anque hoy en día estas tecnologías aún no son implementadas en nuestro país.

\section{PRUEBA DE LABORATORIO PARA LA OBTENCIÓN DE BIOALCOHOLES}

$\mathbf{P}$ ara el estudio técnico preliminar se identificaron unos componentes de la Fracción Orgánica de los Residuos Sólidos Orgánicos (FORSU), que pueden ser transformados a bioetanol mediante procesos y operaciones unitarias. Con el uso de estas etapas se puede llevar a cabo un proceso básico en el que la biomasa se transforma a moléculas de azúcares, las cuales pueden ser fermentadas y desde allí, ser transformadas a alcohol, del cual con su posterior destilación, logrará obtenerse la pureza deseada.

La investigación experimental se llevó a cabo en los laboratorios de la Universidad de Castilla La Mancha (UCLM) en España y se viene replicando en la Universidad EAN (Colombia), en donde se implementó el diseño metodológico de acuerdo con las diferentes fases experimentales requeridas para la evaluación del proceso, con el fin de realizar el análisis al comportamiento de la FORSU mediante procedimientos biológicos y comprobar las probabilidades de la obtención de bioalcoholes además de evaluar los resultados finales.

Durante el proceso global, se llevaron a cabo tres fases experimentales desarrolladas mediante la instalación a escala de un laboratorio de sistema biológico para cada proceso, a saber:

- Adecuación y solubilización de la FORSU.

- Fermentaciones patrón para usar como estándar.

- Fermentación de la FORSU y obtención del bioalcohol. 
Figura 1. Pruebas y puesta en marcha del bioreactor para el desarrollo de las fases del proyecto

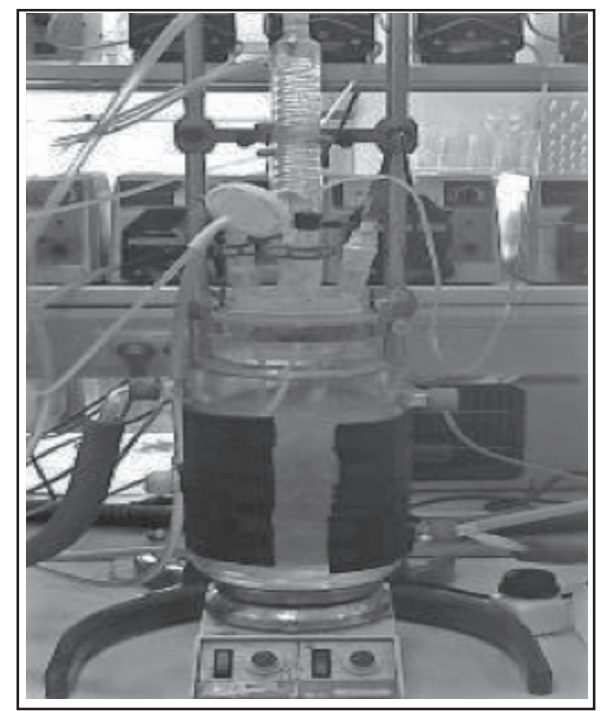

Fuente. Laboratorios UCLM (Ciudad Real, España), 2012.

A continuación se presentan los resultados obtenidos en cada una de las fases experimentales y sus respectivos análisis.

\subsection{Fase 1. Adecuación y solubilización de la FORSU}

Se analizaron los resultados obtenidos de Demanda Química de Oxígeno (DQO) a partir de muestras obtenidas posterior a la adecuación y solubilización (Figura 2).

Figura 2. Variación DQO vs Tiempo

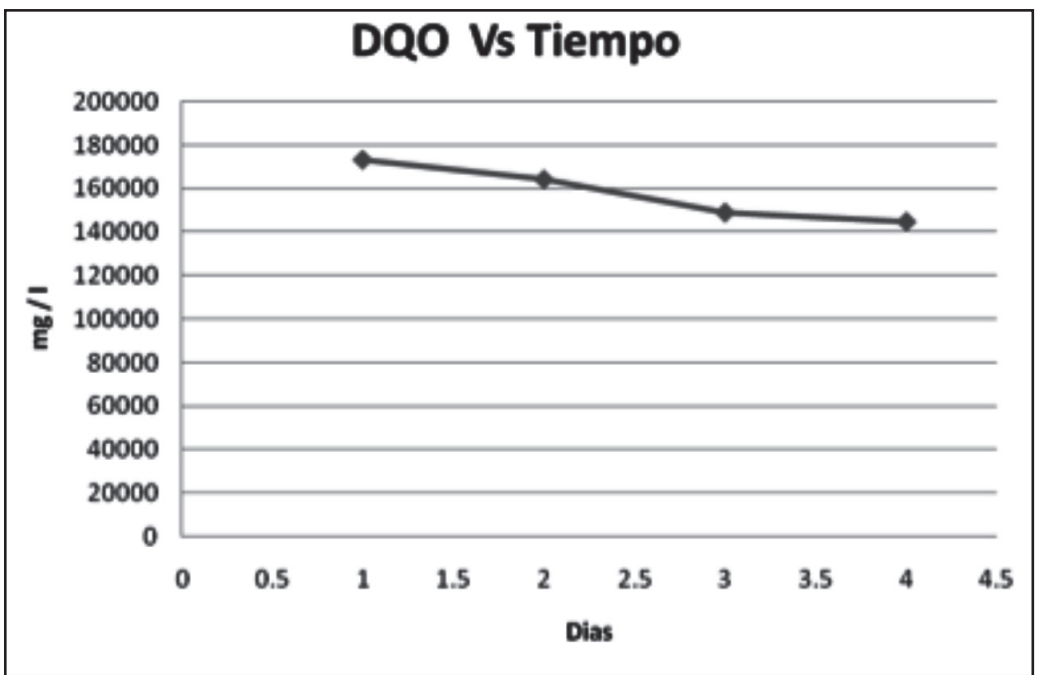

Fuente.Elaboración propia de los autores. 
Los resultados obtenidos de la DQO son valores altos equivalentes al promedio de lo que sería un relleno sanitario jóven (menor a dos años), notándose que posterior al proceso, la solubilización de los compuestos orgánicos fue rápida. Se destaca el descenso de la DQO en el tiempo, lo que podría deberse a transformaciones anaerobias ocurridas durante este proceso, verificándose esta situación con la producción de metano $(\mathrm{CH} 4)$ y de dióxido de carbono $\left(\mathrm{CO}_{2}\right)$ del sistema.

Figura 3. Producción de Gases vs Tiempo

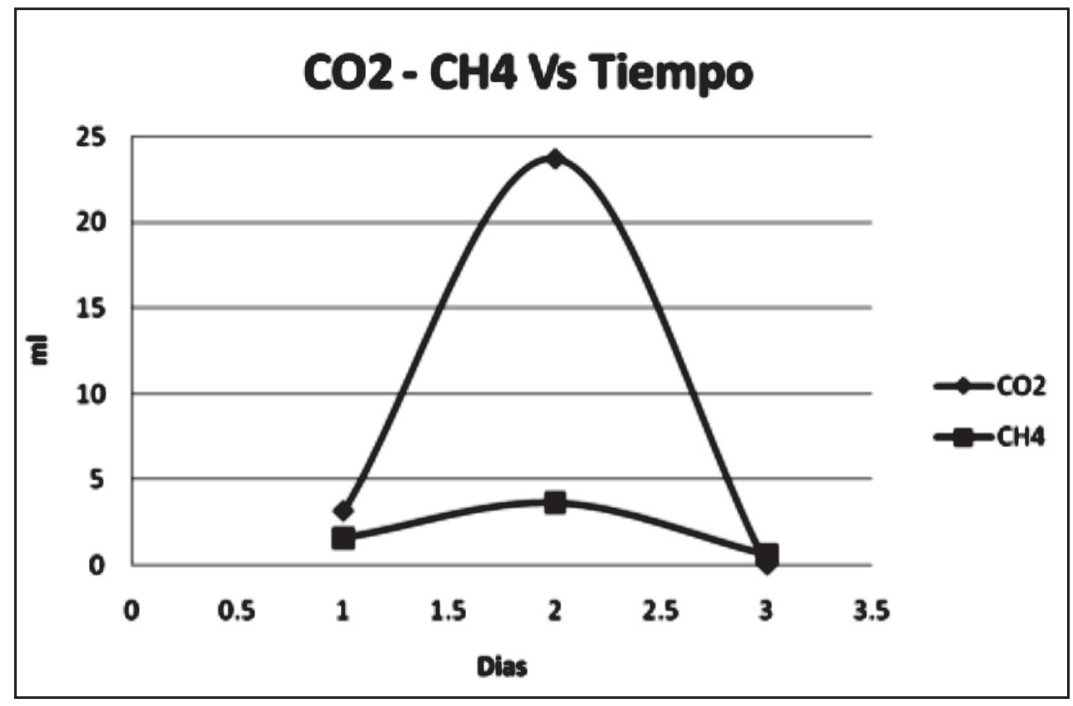

Fuente. Elaboración propia de los autores.

\subsection{Fermentaciones patrón para usar como estándar}

Las medidas de los parámetros convencionales se realizaron de acuerdo con los métodos normalizados de análisis (APHA, 1998), mediante técnicas comúnmente utilizadas en el análisis de aguas. Para el caso de la determinación de los sólidos suspendidos totales (SST) y de los sólidos suspendidos volátiles, se uso el método que se rige por las Normas 2540 D y 2540 E, respectivamente. Así mismo, fueron valoradas las concentraciones de etanol, acetona y glucosa frente al tiempo (Figuras 4 y 5).

Figura 4. Comportamiento de los SST y SSV vs Tiempo
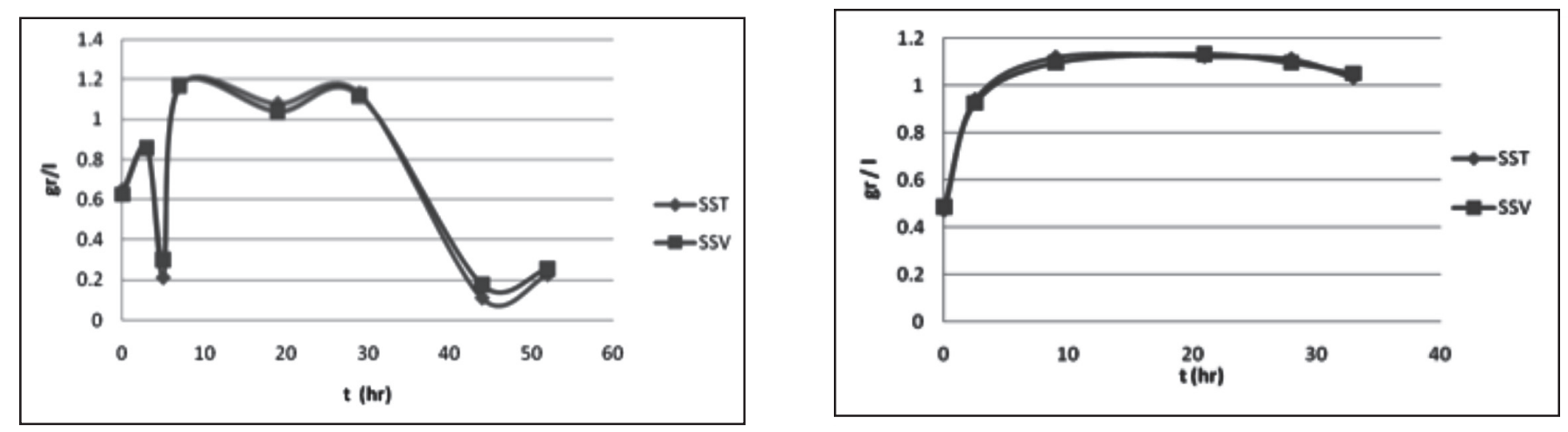

Fuente. Elaboración propia de los autores. 
Figura 5. Producción de bioalcohol, acetona y glucosa vs tiempo

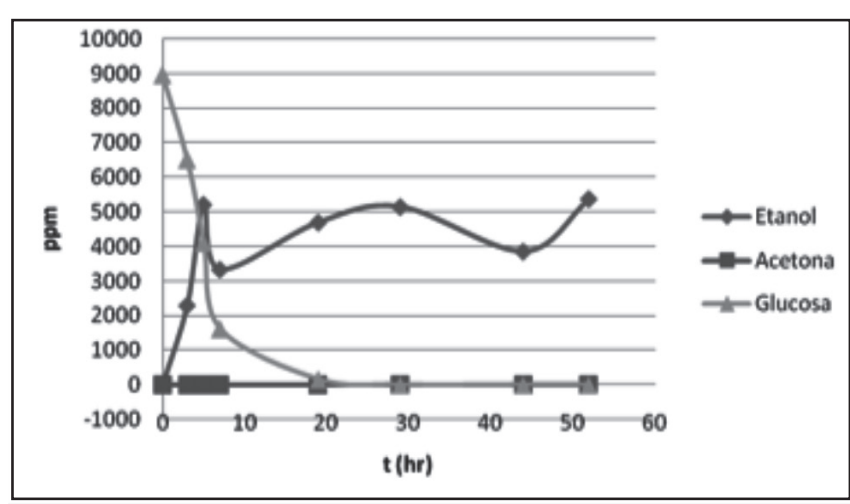

Fuente. Elaboración propia de los autores.

En esta fase, realizados los experimentos sin y con control de pH se observó que la producción de bioalcoholes se podía ver afectada por esta variable, por lo que se decidió incorporarla como una variable de proceso. En términos globales, el rendimiento de etanol alcanzado fue de $0.5 \mathrm{gr}$ etanol $/ \mathrm{gr}$ de glucosa, con producción marginal de $\mathrm{CO}_{2}$ y $\mathrm{CH} 4$.

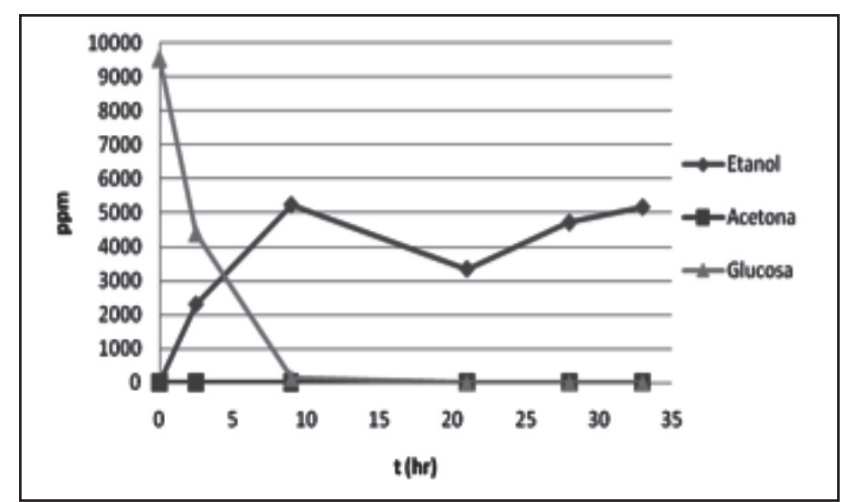

\subsection{Fermentación de la FORSU y obtención del bioalcohol}

Debido a que en esta fase se experimentó con la FORSU ya preparada y solubilizada, se realizó el análisis de la medición de la DQO a partir de cada toma de muestra de manera directa; se presentan los resultados obtenidos de SST y SSV con respecto al tiempo (Figura 6) los resultados de producción de bioalcohol y acetona contra el tiempo (Figura 7).

Figura 6. Comportamiento de los SST y SSV vs Tiempo

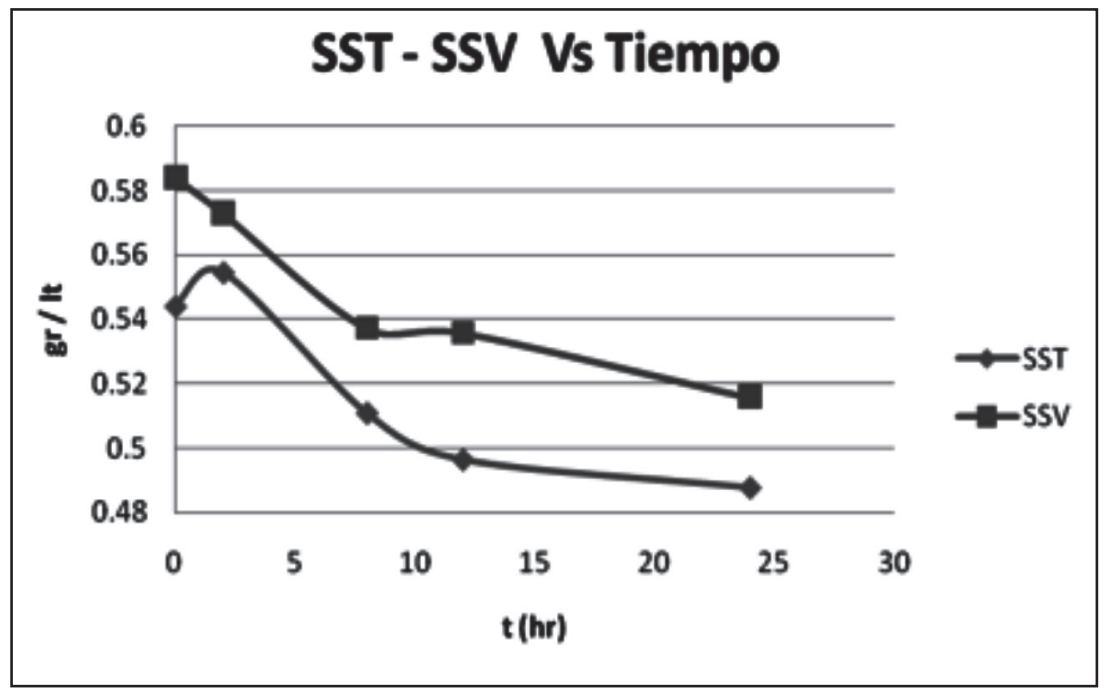

Fuente.Elaboración propia de los autores. 
Figura 7. Comportamiento de la producción de bioalcohol vs Tiempo

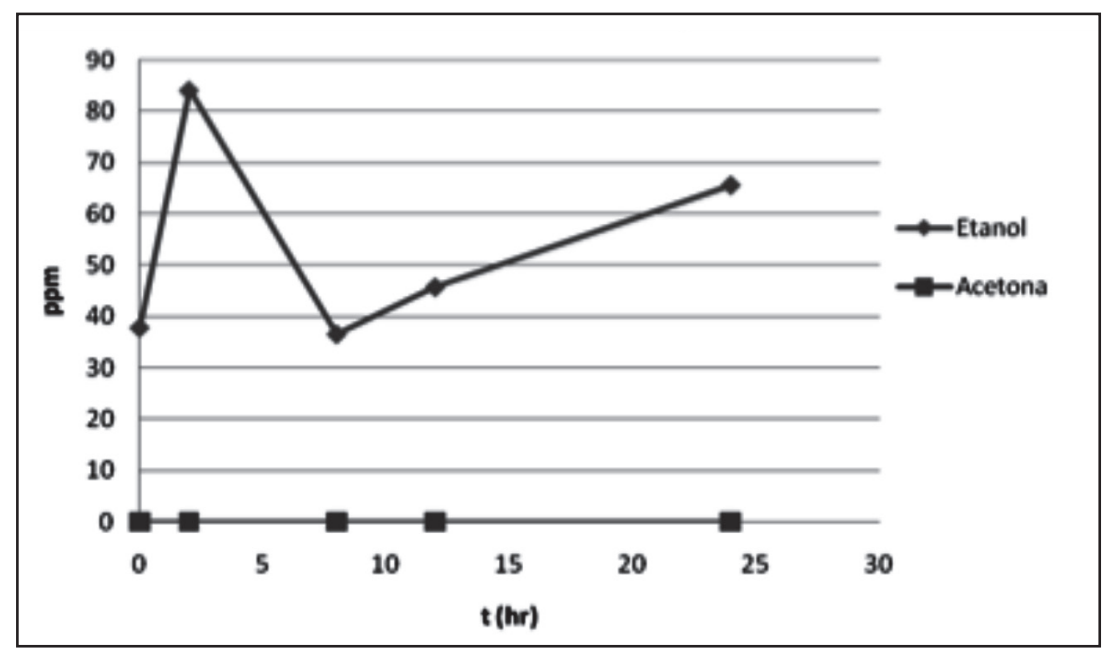

Fuente. Elaboración propia de los autores.

El comportamiento observado para la producción de bioalcohol a través del tiempo en esta fase, se atribuye a una reacción metabólica colateral desarrollada entre las 0 y 7 horas, lo que permitió que se generara como metabolito intermedio el alcohol (o moléculas que podrían dar positivo a los análisis realizados), el cual fue degradado posteriormente y usado como material para producir etanol de forma continua y persistente a partir de la hora 7.
Analizando la evolución de la producción de gases ( $\mathrm{CO} 2$ y CH4), se presentan picos altos y posteriormente valles en su generación, debido a la necesidad de etapas previas de transformación para la fermentación a alcohol. Estos resultados indican que sería necesario aplicar y realizar tratamientos previos, como por ejemplo digestión acida, digestión alcalina u otros métodos que permitan modificar la composición del efluente y facilitar su posterior transformación a alcohol. 


\section{ASPECTOS DE MERCADO Y FINANCIEROS RELATIVOS A LA PRODUCCIÓN INDUSTRIAL DE BIOALCOHOLES EN COLOMBIA}

$\mathbf{P}$ ara realizar un análisis financiero de cualquier proyecto se requieren ciertos datos básicos: el mercado disponible para este y los límites legales impuestos, los gastos operacionales y administrativos y la competencia. Por ello, para este tipo de producción de bioalcohol a base de FORSU, se analizarán estos aspectos en cuanto a mercado y temas financieros.

\subsection{Análisis de mercado}

\subsubsection{Generación de bioetanol en Colombia y el mundo}

La producción de bioetanol en el mundo tiene a algunas naciones como Estados Unidos, Brasil y Europa, como sus principales productores (Figura 8). En Latinoamérica, según una entrevista de América Economía realizada a José Alejandro Martínez, de la Universidad EAN, "...se destacan Brasil, Argentina y Colombia como líderes en la producción de biocombustibles en la región; desde el año 2000, su producción en el mundo ha crecido a un ritmo anual del 10\%, totalizando

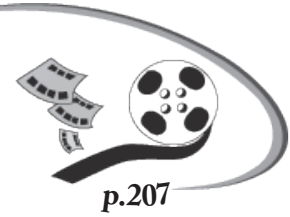
90.187 millones de litros en 2009; de ese total, $82 \%$ corresponde a bioetanol y $18 \%$ a biodiesel. Brasil es el segundo productor de bioetanol del mundo, con 33,2\% de participación en el mercado, después de Estados Unidos (datos de 2009). Colombia, entre tanto, figura en el décimo lugar de países productores, con 0,4\%. Argentina, es el segundo productor mundial de biodiesel, con $13,1 \%$ del mercado, después de Estados Unidos que lidera con 14,3\%; Brasil se ubica en quinto lugar, con 9,7\% de participación...".

Figura 8. Principales productores de bioetanol a nivel mundial

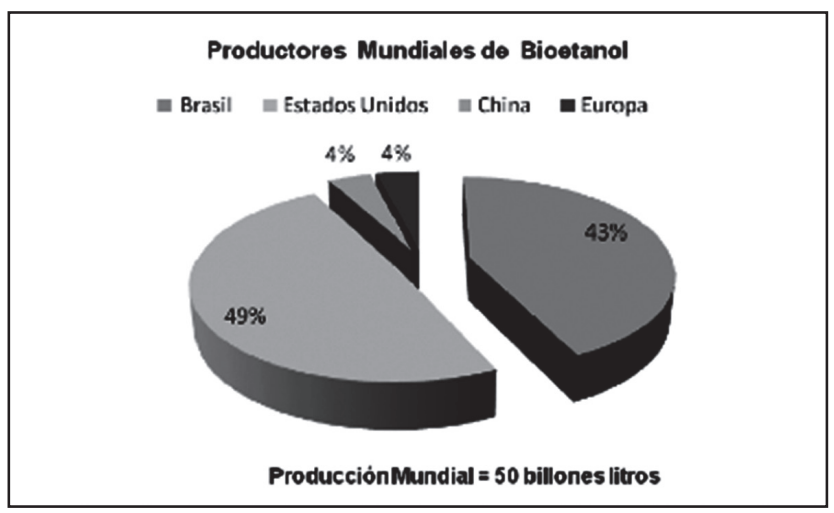

Fuente. Ministerio Federal de Cooperación Económica y Desarrollo de México, 2010.

En Colombia, 19 departamentos consumen mezcla de gasolina con $10 \%$ de etanol, cubriendo el $75 \%$ de la demanda nacional equivalente a aproximadamente 69.000 barriles/día, con posibilidades de incrementar la mezcla a $20 \%$ y el cubrimiento al $100 \%$ del país para el año 2015, según la Federación Nacional de Biocombustibles. A continución se puede observar el histórico de la producción de bioetanol en Colombia en los últimos años (Figura 9). 
Figura 9. Producción histórica de etanol en Colombia

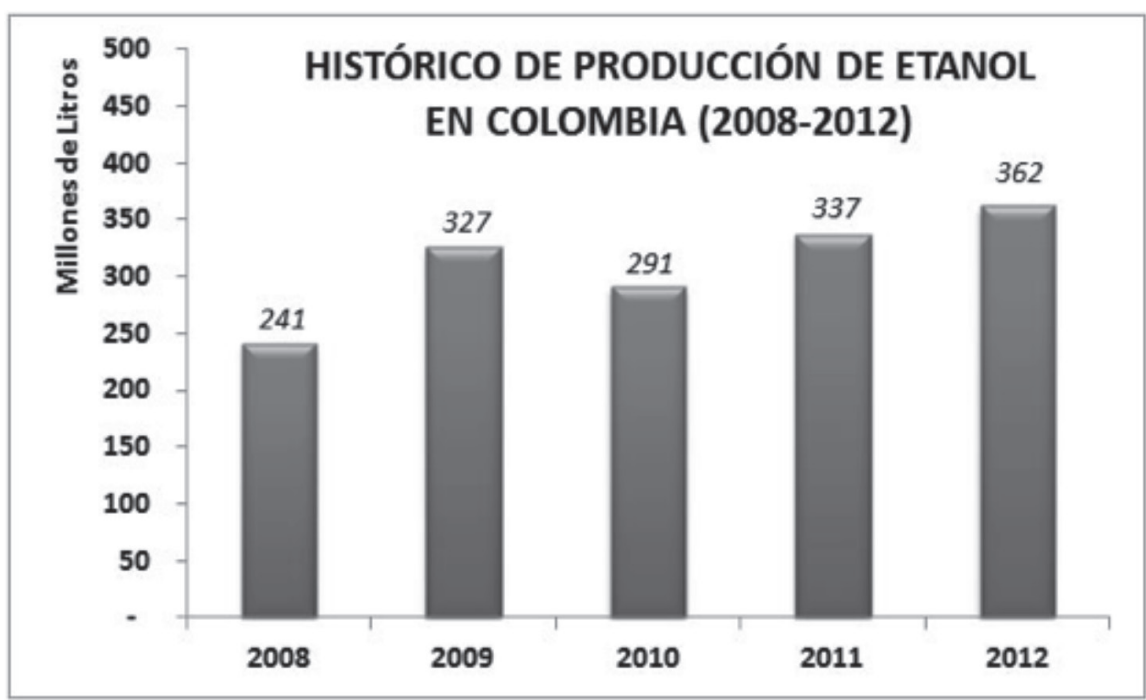

Fuente. Federación Nacional de Biocombustibles, 2012.

En el país, el principal productor de etanol es la industria azucarera, a partir de la caña de azúcar, con cinco plantas en operación (tres en el Valle del Cauca, una en Cauca y otra en Risaralda), que producen en total, un millón de litros/diarios de alcohol carburante (cerca de 6300 barriles/día).

Otros proyectos de producción de etanol se encuentran en desarrollo e investigación: uno para producir 20.000 litros/día a partir de la yuca en Puerto López, Meta. Los ingenios estudian dos plantas más en el Valle, de 150.000 litros/día; también con caña, se estudian dos proyectos en el Meta con capacidad de 400.000 litros/día en total p.207 y uno en Quindío con capacidad de 150.000 litros/día. Entre otros proyectos se encuentra el de Boyacá, la Hoya del Río Suárez (límites Boyacá y Santander), la Costa Atlántica, Antioquia y Tolima.

El mercado objetivo en el país cada vez es más amplio, la alta demanda actual y la poca oferta hacen que el producto tenga óptimas características, bajos costos de producción con relación de otros procesos similares y una creciente demanda por el auge ambiental en el mercado global, de tal forma que favorezca su aceptación en el mercado.

\subsubsection{Consumo de bioetanol en Colombia}

Como se ha mencionado anteriormente, la materia prima son los FORSU y específicamente aquellos ubicados en Bogotá D.C ${ }^{1}$ los cuales ascienden a 6.600 toneladas diarias aproximadamente. De estos el $65 \%$ son Residuos Orgánicos Urbanos lo que da un valor de 4.290 toneladas de posible materia prima en solo la capital.

En temas de consumo según la UPME para 2013 en Colombia hay 8'612.153 vehículos que consumen gasolina, de los cuales el $75 \%$ se encuentran consumiendo 69.000 barriles/día de la mezcla de gasolina con $10 \%$ de alcohol carburante. En Bogotá hay 1'724.643 vehículos que realizan 3'031.669 viajes y todos ellos consumen la mezcla. Actualmente hay un mercado potencial enorme para el futuro, ya que el decreto en proyecto que sustituiría al Decreto 1135 de 2009, aumenta paulatinamente el porcentaje de la mezcla del $10 \%$ al $20 \%$ para gasolina corriente, y del $10 \%$ al $85 \%$ para gasolina extra. Lo anterior, se espera que sea una realidad para el 2030. Así mismo, se busca la eliminación de la restricción actual de los departamentos en los que obligatoriamente debe estar la mezcla y en los que no².

\footnotetext{
${ }^{1}$ Esto por tener el $26 \%$ de FORSU del País, y dando la ventaja que en la mayoría se destina al Relleno Sanitario Doña Juana.

${ }^{2}$ Como se comentó anteriormente, la mezcla solo está en 19 departamentos de Colombia.
} 
Adicionalmente al consumo anteriormente descrito, se puede observar que los bioalcoholes tienen otros mercados potenciales como las chimeneas (quemadores), que se utilizan principalmente para calefacción de ambientes. Según precios comerciales en el medio, un galón de bioetanol producido a partir de caña de azúcar, se vende en $\$ 24.000$ (poco más de 12 USD) y cuenta con un $96 \%$ de pureza. En una chimenea (quemador) convencional, 1.5 litros pueden quemarse en cuatro horas aproximadamente y tienen un poder calorífico de 2.500 y 5.500 vatios, sin embargo, al ser un mercado no regulado no hay cifras ciertas de consumo actual para ahondar en la demanda específica y sus proyecciones.
El mercado alterno se divide así: de un 100\% de consumo de gasolina en Colombia, el 3.6\% va para el sector agroindustrial; $1.6 \%$ es para el sector residencial y generación de energía eléctrica y $2.9 \%$ para el sector industrial, comercial y construcción (UPME, 2012). El restante 91.9\% es para vehículos, por lo que los mercados alternos para el bioetanol aún no son representativos para el presente proyecto.

Figura 10. Distribución porcentual del consumo de gasolina en Colombia

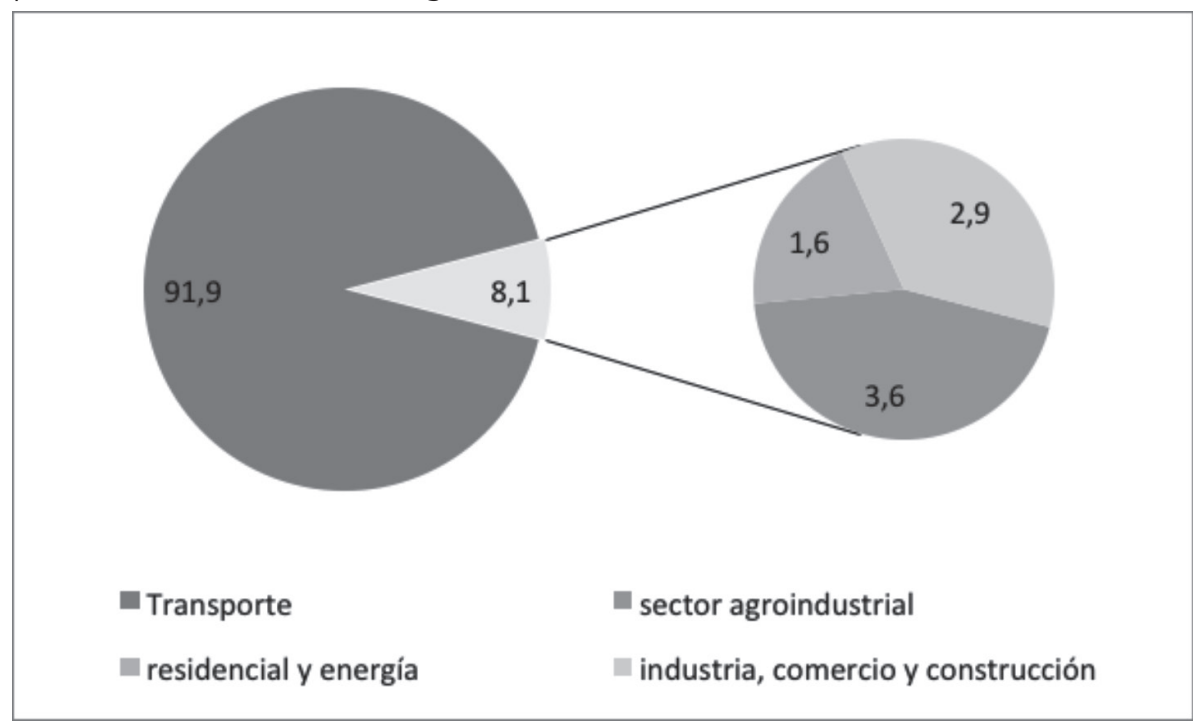

Fuente. Elaboración propia de los autores, 2014.

\subsection{Análisis financiero}

Para iniciar se requiere lo básico: una planta, instalaciones, terreno, etc. A esto se le adicionan los gastos administrativos, gastos operacionales, gastos legales, entre otros y finalmente se realizan proyecciones. Todo esto tomando en cuenta el proceso químico explicado anteriormente.

Los supuestos básicos para la presente planeación financiera ${ }^{3}$ se muestran a continuación (Tabla 1):

3 El precio por litro de bioetanol es regulado mensualmente por el Estado y se publica de forma oficial por Fedebiocombustibles. Las proyecciones se ajustan a esta variación a través de un promedio de las variaciones presentadas en los últimos cinco años. 
Tabla 1. Supuestos básicos para la planeación financiera

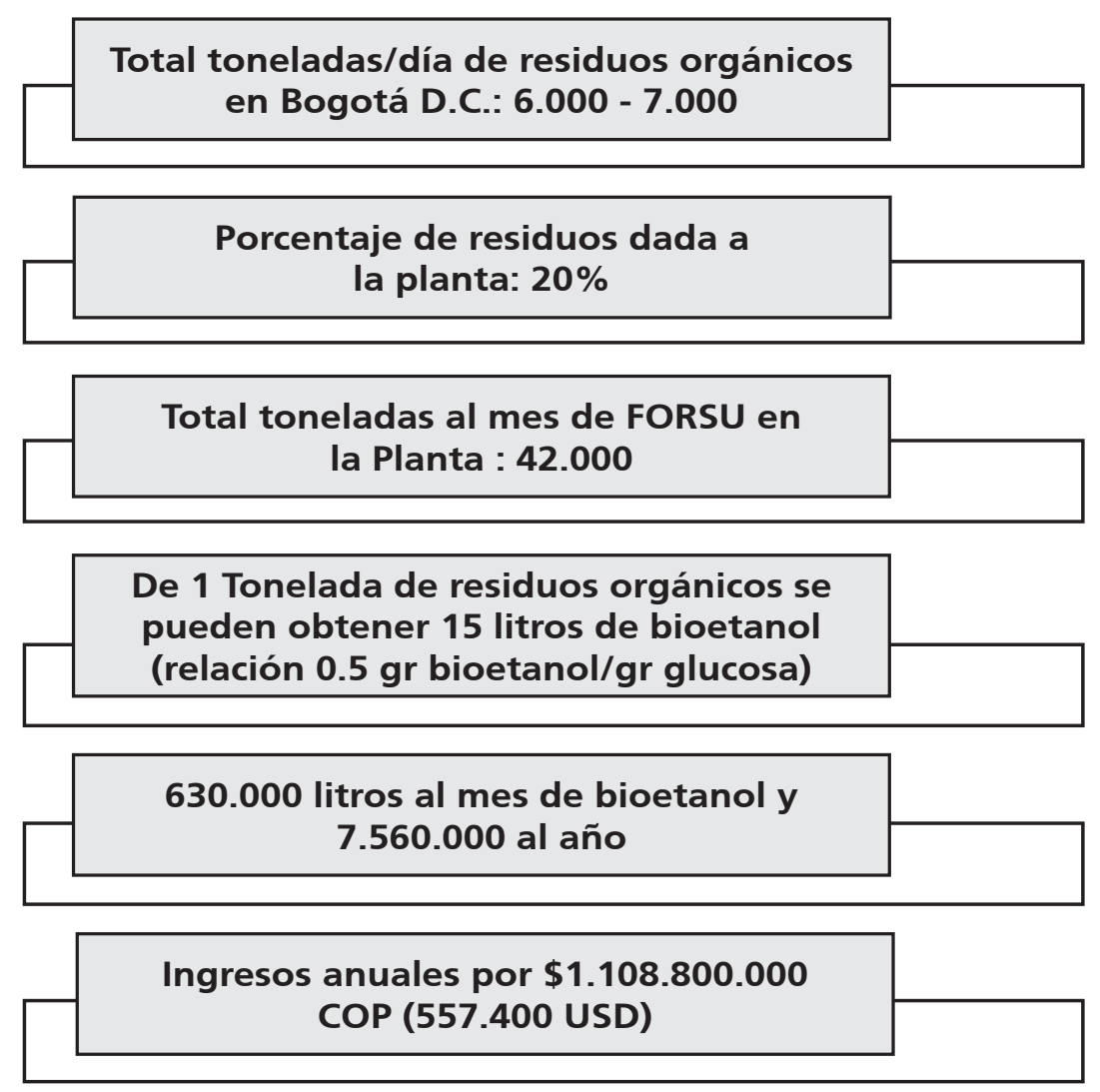

Fuente. Elaboración propia de los autores.

La planta ubicada en las cercanías del Relleno Sanitario Doña Juana, requeriría un terreno de $3.857 \mathrm{~m}^{2}$, en donde el metro cuadrado tiene un valor de $\$ 700.000$, para un total de $\$ 2.700 .000 .000$ (1.350.000 USD) por concepto de la adquisición del terreno de la planta. La maquinaria de la planta, con todos los equipos y tubería requeridos, se cotiza en USD4.100.000 que a una tasa de cambio de $\$ 2.000$ da un total de $\$ 8.200 .000 .000$. Hay que adicionar el valor del montaje y construcción, el cual tiene un valor aproximado de $\$ 2.460 .000 .000$; a esto se le agrega la construcción de la planta administrativa, lo que genera un gran total para la inversión inicial de $\$ 14.000 .000 .000$ (USD 7’000.000) aproximadamente.
Se deben tener en cuenta las disposiciones legales de inventario, los gastos operacionales y los costos de producción que inciden en las cifras; por ejemplo los inventarios que por disposición legal, se requiere de diez días de inventario disponibles siempre en el patio de acopio; se agregan dos días para inventario en proceso de rotación y cinco días para la materia prima en rotación.

La evaluación financiera resumida se muestra a continuación ${ }^{4}$ (Tabla 2).

\footnotetext{
${ }^{4}$ La base son los supuestos que arrojó el análisis de prefactibilidad realizado por línea de investigación en gestión de residuos sólidos de la Universidad EAN en 2013.
} 
Tabla 2. Evaluación financiera

\begin{tabular}{|c|c|}
\hline \multicolumn{2}{|c|}{$\begin{array}{c}\text { Evaluación del proyecto Planta de Bioalcohol } \\
\text { a Base de FORSU }\end{array}$} \\
\hline Tasa mínima de rendimiento & $18 \%$ \\
\hline TIR (Tasa Interna de Retorno) & $29,41 \%$ \\
\hline VAN (Valor actual neto) COP & 7.117 .008 .862 \\
\hline PRI (Periodo de recuperación de la inversión) & 2,50 \\
\hline $\begin{array}{l}\text { Duración de la etapa improductiva del } \\
\text { negocio ( fase de implementación) }\end{array}$ & 8 meses \\
\hline $\begin{array}{l}\text { Nivel de endeudamiento inicial del negocio, } \\
\text { teniendo en cuenta los recursos del fondo } \\
\text { emprender. ( AFE/AT) }\end{array}$ & $50,00 \%$ \\
\hline $\begin{array}{l}\text { Periodo en el cual se plantea la primera } \\
\text { expansión del negocio ( Indique el mes) }\end{array}$ & 15 meses \\
\hline $\begin{array}{l}\text { Periodo en el cual se plantea la segunda } \\
\text { expansión del negocio }\end{array}$ & 16 meses \\
\hline
\end{tabular}

Fuente. Elaboración propia de los autores, 2014.

El análisis financiero fue hecho en un escenario medio, en donde se manejaría una cantidad del $20 \%$ del total de los residuos orgánicos de Bogotá, con aportes del $50 \%$ de los socios y $50 \%$ deuda; el total de litros producidos, se vendería al distribuidor mayorista. También se asume que el precio del litro de etanol, se incrementará con la inflación y el índice de precios. Si se cumplieran estos supuestos se lograría a diez años una TIR de $29.41 \%$ y un VAN de siete mil millones de pesos, una tasa de improductividad de ocho meses, y una posible expansión del negocio año y medio después de dar utilidades. 


\section{CONCLUSIONES}

- El aprovechamiento de los residuos sólidos orgánicos genera un alto impacto ambiental positivo en países en vías de desarrollo por lo tanto, la contribución en el manejo de estos residuos para generar productos de valor agregado permitirá generar opciones de aprovechamiento que aporten al desarrollo no dependiente de hidrocarburos de estos países.

- Los residuos sólidos orgánicos son abundantes en nuestra región latinoamericana, representando la fracción de mayor porcentaje de RSU. Tan solo una mínima parte de ellos es empleada y valorizada; por ello, es vital desarrollar nuevos procesos y crear nuevas cadenas productivas.

- Actualmente, el mercado de bioetanol está siendo derivado desde la caña de azúcar como materia principal, así como desde el almidón de maíz, ambos insumos que pueden afectar la sostenibilidad alimentaria de los territorios, o afectar por vía demanda, el precio de los alimentos; el buscar nuevas formas de producir bioalcoholes sin afectar la seguridad alimentaria, es una necesidad para generar energías alternativas.

- A través del proyecto de investigación desarrollado en la Especialización en Gestión de Residuos Sólidos de la Universidad EAN (Colombia), se pudo llevar a cabo un proceso en el cual fue posible obtener por vía fermentativa bioalcohol a partir de la FORSU con un rendimiento aceptable $(0,5 \mathrm{gr}$ bioalcohol/gr de Glucosa); sin embargo, la FORSU está constituida por moléculas complejas que hacen necesario el análisis que permitan transformaciones previas a la fermentación para hacer disponibles los componentes principales y obtener mejores resul-tados y rendimientos.

- Para este proceso, a nivel industrial no existe competencia instalada directa en términos de la misma tecnología a nivel nacional y hay un mercado sin explotar, por lo que las ventajas competitivas son visibles y aptas para el desarrollo financiero, siempre y cuando se tenga una acción conjunta con la alcaldía local para un constante flujo de insumo. Sin embargo, existen proyectos que se están desarrollando y que a partir de otros insumos, buscan obtener el mismo producto.

- A primera vista, es un proyecto rentable, con una evaluación financiera óptima y con grandes miras a expansión, pero existen muchas variables que han de ser consideradas si se desea un análisis más profundo. En general, sería un muy buen proyecto en el cual vale la pena invertir pero se resalta que al ser un proyecto de tan alta envergadura, se debe hacer, un análisis financiero que incluya más variables. 


\section{REFERENCIAS BIBLIOGRÁFICAS}

Betancourt C., Martínez S. (2012). Analysis of use of leachate from a landfill in an intermediate city in Colombia. Memorias del XXVII Congreso Centroamericano de Ingeniería Sanitaria y Ambiental. AIDIS El Salvador.

Cinzia B., Francesco F. (2011). Anaerobic digestion of mechanically treated of MSW: Experimental data on biogas/methane production and residues characterization. Italia:University of Perugia, Biomass Research Centre (CRB).

Martin J., Potts L. and Reeves A. (1997). Small-scale simulation of waste degradation in landfills (s.c): Chapman \& Hall.

Martínez, S. (2012). Propuesta para el desarrollo de un spin off en residuos sólidos en Bogotá - Colombia. Revista Ciencia y Tecnología del Ejército. pp. 64-75.

Martínez, S. (2013). Use and valorization of Organic Fraction of municipal solid waste in Colombia for sustainable development. Revista Ontare. pp. 243 a 254. Bogotá: Universidad EAN.

Montoya G. (2012). Análisis de la Viabilidad de obtención de bioetanol a partir de la Fracción Orgánica de los Residuos Sólidos Urbanos (FORSU). España: Universidad Castilla La Mancha; Bogotá: Universidad EAN.

Sierra R., Diaz R. (2014). Estudio de Prefactibilidad de una planta de transformación de Residuos Orgánicos en Bioetanol (PTROE), Bogotá D.C., Colombia. Recuperado de: http://1drv.ms/NnInrV

UAESP. (2012). Diagnóstico Residuos Sólidos dispuestos en el Relleno Sanitario Doña Juana, Bogotá (s.c). 


$$
F
$$

